\section{NEONATAL MORBIDITY AND MORTALITY IN EXTREMELY PRETERM SMALL FOR GESTATIONAL AGE INFANTS}

doi:10.1136/archdischild-2012-302724.1277

${ }^{1} \mathrm{~K}$ Itabashi, ${ }^{1 T}$ Yamakawa, ${ }^{2} \mathrm{~S}$ Kusuda, ${ }^{3} \mathrm{M}$ Fujimura. ${ }^{1}$ Pediatrics, Showa University School of Medicine; ${ }^{2}$ Tokyo Women's Medical University; Tokyo; ${ }^{3}$ Osaka Medical Center and Research Institute for Maternal and Child Health, Osaka, Japan

Background Neonatal mortality and morbidity in extremely preterm infants ( $<28$ weeks of gestation) have been extensively studied, but the risk added by intrauterine growth restriction remains controversial.

Aim To assess whether intrauterine growth restricted (small for gestational age, SGA) extremely preterm infants show a further increase in neonatal mortality and morbidity.

Methods The study included 9,888 singleton extremely preterm infants whose live birth was recorded at the Neonatal Research Network in Japan during 2003-2010. SGA was defined as birth weight at least 2SD below the mean for gestational age. The Risk of mortality and morbidity in the SGA group was evaluated by comparing outcomes for SGA against a non-SGA reference group.

Results Of the study subjects, 1,215 (12.3\%) were SGA. Controlling for gestational age, sex, parity and multiple gestation, SGA infants showed a higher mortality rate during NICU stay compared with reference group infants (odds ratio [OR]: 4.23, $\mathrm{p}<0.0001$ ). Severe neonatal asphyxia (OR: $1.89, \mathrm{p}<0.0001)$, RDS (OR: 1.33 , $\mathrm{p}<0.0001)$, chronic lung disease at 36 weeks' postmenstrual age (OR: 2.23, $\mathrm{p}<0.0001$ ), sepsis (OR: 1.95, $\mathrm{p}<0.0001$ ), necrotizing enterocolitis (OR: 1.93, p<0.0001), focal intestinal perforation (OR: $1.46, \mathrm{p}=0.011$ ) and congenital anomalies (OR: 2.66, $\mathrm{p}<0.0001)$ were significantly associated with SGA status.

Conclusion Extremely preterm SGA infants are associated with increased the risk of neonatal mortality and major morbidity. These results are important for obstetric counseling and decision making and treatment of extremely preterm infants.

\section{OUTCOME FOLLOWING FETAL PLEUROAMNIOTIC SHUNTING IN 114 LARGE HYDROTHORACES}

doi:10.1136/archdischild-2012-302724.1278

${ }^{1} \mathrm{E}$ Kelly, ${ }^{2} \mathrm{G}$ Seaward, ${ }^{2} \mathrm{R}$ Windrim, ${ }^{2} \mathrm{G}$ Ryan. ${ }^{1}$ Paediatrics; ${ }^{2}$ Obstetrics \& Gynecology, Mt Sinai Hospital, Toronto, ON, Canada

Introduction Untreated, fetal hydrothorax is associated with significant morbidity and a mortality rate of approximately $80-90 \%$.

Population 114 fetuses with isolated large pleural effusions underwent pleuroamniotic shunting at our perinatal centre. All had an extensive antenatal work-up including: detailed anatomy, echocardiogram, karyotype, infectious testing for CMV, Toxoplasmosis and Parvovirus. 84 were bilateral, 72 (63\%) were hydropic and 41 (36\%) had associated polyhydramnios,. Mean maternal age was 30.2 years, the mean gestation at diagnosis was 25 weeks and at shunting 27.6 weeks. 64 required bilateral shunts and, of unilateral, 27 were leftsided and 23 right-sided. 25 underwent simultaneous amnioreduction. The mean interval to delivery was 7 wks.

Results There were 15 (12.3\%) intrauterine deaths. 26 (23\%) neonatal deaths and $73(64.7 \%)$ survivors. Additional abnormalities including genetic, metabolic and neurological syndromes were identified antenatally in 8 cases and postnatally in 9. Of 99 liveborn babies, $76(77 \%)$ delivered at our perinatal centre. Postnatally, 46 required ventilation, $38(50 \%)$ required chest tubes, of whom 19 $(26 \%)$ died. Of 73 survivors, 2 (3\%) were lost to follow-up, $4(5.5 \%)$ are $<4$ months of age, 10 (14\%) showed evidence of significant developmental delay, including 3 with Trisomy 21, and 1 had mild developmental delay. Fifty five (75\%) are developing normally.

Conclusion Fetal hydrothorax can be associated with a wide range of conditions, some of which may not be detectable antenatally.
Fetal therapy significantly improves perinatal outcome, although mortality remains high. On long term follow up, approximately $75 \%$ of survivors are developmentally normal.

\section{EARLY OUTCOMES FOLLOWING REFERRALS FOR THERAPEUTIC HYPOTHERMIA - A REGIONAL NEONATAL TRANSFER SERVICE PERSPECTIVE}

doi:10.1136/archdischild-2012-302724.1279

1.2PK Yajamanyam, ${ }^{2} \mathrm{~S}$ Mohinuddin. ${ }^{\top}$ Neonatal Unit, Birmingham Women's Hospital NHS Foundation Trust, Birmingham; ${ }^{2}$ Neonatal Transfer Service, Barts Health NHS Trust, London, UK

Background and Aims Therapeutic hypothermia $(\mathrm{TH})$ is now a standard of care for neonatal encephalopathy (NE). We have previously shown that referrals for $\mathrm{TH}$ in the London region have steadily increased since the publication of TOBY study but documentation of cooling criteria was poor (43\%) before transfer to cooling centres. In this study we audit referrals for $\mathrm{TH}$ following introduction of a structured proforma and the early outcomes of these babies.

Methodology Prospective audit of referrals for TH to a regional neonatal transfer service over a six-month period (May-October 2011). Audit registered with the Clinical Effectiveness unit of the NHS Trust. Following transfer, cooling centres was contacted to find out early outcomes: if infants received $\mathrm{TH}$ for 72 hours and outcome at 7 days.

Results 43 referrals for $\mathrm{TH}$ were received. The median Gestation was 40(35-42) weeks, Birth-weight 3.42(2.04-4.84) Kg. Of these 38 transfers were performed. Completed proforma was available in 21 cases. TOBY criteria A were recorded in $100 \%$ of cases and TOBY criteria B in $88 \%$. $8(21 \%)$ infants did not receive $\mathrm{TH}$ for 72 hours as assessed to not benefit from $\mathrm{TH}$ of which 3 died within 72 hours. At 7 days of age 5 were discharged home and remaining 30 were inpatients.

Conclusions Our audit shows that introduction of a structured proforma can improve documentation of cooling criteria and neurological examination. We recommend that any referral for $\mathrm{TH}$ is carefully selected to avoid unnecessary transfer of neonates who may not benefit from $\mathrm{TH}$.

\section{EFFECTS OF AMBIENT NOISE ON COCHLEAR FUNCTIONS IN NEWBORNS GRADUATED FROM NICU}

doi:10.1136/archdischild-2012-302724.1280

S Beken, E Önal, B Gündüz, Y Kemaloğlu, U Çakır, M Özden, I Karagöz, F Kulalı, i Hirfanoğlu, C Türkyılmaz, E Ergenekon, E Koç, Y Atalay. Gazi University Hospital, Ankara, Turkey

Background and Aim Neonatal Intensive Care Unit (NICU) is a noisy environment in which infants can be exposed to high noise levels. The aim of the study is to evaluate the adverse effects of noise on hearing and, neurological outcomes of NICU graduates at six months of age.

Methods Thirty two infants that had been admitted to Gazi University Hospital NICU and 25 healthy controls, were included in the study. Noise levels were recorded continously during hospitalization period. TEOAE, DPOAE and ABR tests were used to assess hearing. Neurological outcome was assessed with Bayley II Infant Development Scale.

Results The median period of noise exposure above $45 \mathrm{~dB}$, was $50.1 \%$ of the entire hospitalization period. Levels exceeding $45 \mathrm{~dB}$ were mostly below $124 \mathrm{~Hz}$. Major source of noise was traced back to the incubators. All patients passed the hearing screening tests before discharge. On the sixth month follow up; hospitalized infants had lower DPOAE SNR amplitudes $(\mathrm{dB})$ at five frequencies including 1001, 1501, 3003, 4004, $6006 \mathrm{~Hz}$ in both ears. DPOAE fail rates at $1001 \mathrm{~Hz}$ and $1501 \mathrm{~Hz}$ were higher in hospitalized infants 